\title{
Efectos de un programa de entrenamiento de fuerza sobre la composición corporal y la fuerza máxima en jóvenes entrenados
}

\author{
Francisco javier Orquín Castrillón ${ }^{a}$, Gema Torres-Luque ${ }^{\mathrm{b}}$ y Facundo Ponce de Leónc \\ aDepartamento de Ciencias de la Actividad Física y del Deporte. Universidad Católica San Antonio. Murcia. España. \\ bFacultad de Humanidades y Ciencias de la Educación. Universidad de Jaén. Jaén. España. \\ Instructor de Salas de Musculación. Buenos Aires. Argentina.
}

\section{RESUMEN}

El objetivo de este estudio fue conocer las adaptaciones en composición corporal, fuerza máxima y resistencia muscular local de un programa de entrenamiento de fuerza en circuito intermitente con sobrecargas.

Método: Se seleccionaron 8 varones jóvenes entrenados en musculación $(24,07 \pm 3,12$ años, 77,96 $\pm 7,5 \mathrm{~kg}, 173 \pm$ $0,2 \mathrm{~cm}$ y $14,50 \pm 2,00$ meses de experiencia). Todos los sujetos firmaron un consentimiento por escrito de participación. Se realizó una valoración antropométrica, 10 tests de una repetición máxima (IRM) y un test de resistencia muscular local. Posteriormente se llevó a cabo un programa de entrenamiento de fuerza de 8 semanas de duración con la característica de ser en circuito intermitente con sobrecargas, 3 veces por semana, entre el 62 y el $72 \%$ de IRM. Pasado este tiempo se realizó una valoración final con las mismas características de la valoración inicial.

Resultados: Los resultados mostraron una disminución en los porcentajes de grasa corporal en torno al 9,46\%, mejoras en fuerza máxima en torno al 3-31\% según el grupo muscular, $y$ un aumento en la resistencia muscular local del I,42\%.

Conclusiones: El entrenamiento en circuito intermitente con sobrecargas tras 8 semanas de duración ofrece adaptaciones a nivel de composición corporal, de fuerza máxima y resistencia muscular local para sujetos entrenados. Aunque se requieren estudios con muestras más amplias, se presenta como una alternativa de metodología dentro de la sala de musculación.

PALABRAS CLAVE: Entrenamiento de fuerza. Entrenamiento en circuito. Composición corporal.

\section{ABSTRACT}

The aim of this study was to know the adjustments in corporal composition, maximum force and muscular local resistance of a program of training of force in intermittent circuit with overcharges.

Method: Eight young males trained in resistance $(24,07 \pm$ 3,12 years, $77,96+7,5 \mathrm{~kg}, 173 \pm 0,2 \mathrm{~cm}$ and $14,50 \pm 2,00$ months of experience) were selected. All the subjects signed an assent in writing of participation in the same one. Characteristic anthropometric, I0 tests of IRM and one test of muscular local resistance was realized. Later, there was carried out a program of resistance training of 8 weeks of duration by the characteristic of being in intermittent circuit with overcharges, 3 times per week, between 62\%$72 \%$ of IRM.A final valuation with the same characteristics of the initial valuation was realized.

Results: The results showed a decrease in the percentages of corporal fat of $9,46 \%$, improvements in maximum strength about $3 \%-31 \%$ and an increase in the muscular local resistance of I,42\%.

Conclusions: The training in intermittent circuit with overcharges after 8 weeks of duration offers adjustments to level of corporal composition, of maximum strength and muscular local resistance for trained subjects.

KEY WORDS: Strength Training. Circuit Weight Training. Body Composition. 


\section{INTRODUCCIÓN}

Existen numerosos métodos y ejercicios para obtener adaptaciones en las diferentes capacidades físicas. En la actualidad están cobrando una mayor importancia los métodos relacionados con la movilización de sobrecargas (pesas), ya que son métodos muy eficaces al ofrecer adaptaciones muy interesantes a un amplio campo de poblaciones de diferentes edades y características. Aunque los entrenamientos de fuerza producen determinadas mejoras neuromusculares, se suelen prescribir por sus beneficios relacionados con los aumentos de fuerza muscular y los efectos sobre la composición corporal.

Entre las adaptaciones más relevantes sobre la composición corporal destaca el incremento del tamaño de la sección transversal de las fibras musculares (hipertrofia muscular) ${ }^{1-3}$, la mejora de la densidad mineral ósea (DMO), especialmente en los lugares cercanos a los músculos que intervienen en el ejercicio ${ }^{4}$, así como el aumento de la calidad del hueso, haciéndolo más resistente ${ }^{5}$ y la disminución de los valores de masa grasa corporal ${ }^{2}$.

Los efectos beneficiosos del entrenamiento con sobrecargas dependen de la manipulación de numerosos factores, como la intensidad de la carga, el volumen y la frecuencia de entrenamiento, el tiempo de recuperación y la velocidad de movimiento, que según los objetivos y metas que se pretendan alcanzar se modifican de una forma u otra ${ }^{6-9}$. Aunque las variables del entrenamiento están bastante estudiadas y han sido identificadas, aún hoy existe incertidumbre en cuanto a la relativa importancia y especificidad de cada una de ellas sobre el desarrollo de la fuerza y la ganancia de hipertrofia muscular ${ }^{1}$. Aun así, la intensidad y el volumen del entrenamiento se consideran las variables principales a modificar en este tipo de entrenamientos ${ }^{6}$.

Para conseguir mejoras en fuerza máxima y en composición corporal se pueden utilizar diferentes métodos de entrenamiento. De hecho, hay gran documentación sobre mejoras en estas cualidades con el uso de entrenamientos contra resistencias en circuito (circuit weight-training) en sujetos desentrenados. Estudios del entrenamiento en circuito de 12 semanas de duración, con intensidades comprendidas entre el 20 y el $40 \%$ de una repetición máxima (1RM), producen aumentos en fuerza del 2 al 17\% y las adaptaciones obtenidas en los porcentajes de grasa son de una disminución hasta del 3 al $4 \%{ }^{10,11}$. En programas de 10 semanas de duración utilizando intensidades de entrenamiento entre el 40 y el $60 \%$ de 1 RM se observa que se obtienen mejoras en fuerza máxima comprendidas entre el 6 y el $42 \%$. Por otro lado, se obtienen disminuciones de hasta $2,6 \%$ en los porcentajes de grasa corporal ${ }^{12-14}$.

Si se analizan los estudios de 8 semanas de duración con unas intensidades en torno al $40 \%$ de $1 \mathrm{RM}$, se observan incrementos del 5 al 32\% en fuerza máxima y una disminución comprendida entre el 2 y el 4,3\% en los porcentajes de grasa corporal $^{15,16}$. Estos estudios indican que con programas de corta duración (entre 8 y 12 semanas) se consiguen mejoras significativas en fuerza máxima (entre el 2 y el 42\%) y en la composición corporal, exactamente en la disminución de los porcentajes de grasa corporal (hasta un 4,3\%) ), $11,12,17-19^{\text {. }}$

El presente estudio tiene como objetivo conocer los efectos de un programa alternativo de fuerza mediante un circuito intermitente con sobrecargas, de 8 semanas de duración, sobre la composición corporal y la fuerza máxima en sujetos experimentados en el entrenamiento de musculación.

\section{Método}

\section{Muestra}

La muestra estuvo formada por 8 varones sanos $(24,07 \pm$ 3,12 años, $79,7 \pm 7 \mathrm{~kg}, 173 \pm 0,2 \mathrm{~cm}$ y $14,50 \pm 2,00$ meses de experiencia en la sala de musculación) cuyas características se ofrecen en la tabla I. Todos los sujetos fueron informados acerca de los procedimientos, riesgos y beneficios del estudio, y firmaron un informe de consentimiento previo al comienzo del mismo. Para seleccionar a los sujetos experimentales se realizó un muestreo intencional opinático, ya que los sujetos debían cumplir con los siguientes requisitos: ser varón joven (20-30 años), no presentar ningún tipo de patología o lesión incompatible con los entrenamientos y ser considerado experto en trabajo de musculación, que según Carpenilli et $\mathrm{al}^{20}$ son los que movilizaban más del $100 \%$ de su peso corporal en el ejercicio de press de banca y de más del 130\% en el ejercicio de prensa

Tabla

Características de los individuos de la muestra

\begin{tabular}{|llllll|}
\hline Sujetos & Edad (años) & Peso $(\mathbf{k g})$ & Talla $(\mathbf{m})$ & IMC & Experiencia (meses) \\
\hline 8 & $24,07 \pm 3,12$ & $77,96 \pm 7,5$ & $1,73 \pm 0,2$ & $25,82 \pm 2,47$ & $14,50 \pm 2,00$ \\
\hline
\end{tabular}

IMC: índice de masa corporal. 
de piernas, así como una experiencia en el entrenamiento con sobrecargas superior a un año.

Por otro lado, los criterios o factores de exclusión del estudio fueron: consumir cualquier tipo de suplementación (proteínas, creatina, L-carnitina, hidratos de carbono, aminoácidos) o sustancias dopantes relacionadas con el rendimiento físico (esteroides, hormonas), realizar otra actividad física que pudiese influir en los resultados del estudio, no respetar las pautas de entrenamiento dictadas en el estudio y no faltar a dos o más sesiones de entrenamiento.

\section{Procedimiento}

Los sujetos experimentales realizaron una valoración inicial, un programa de entrenamiento de 8 semanas de duración y una valoración final. La evaluación inicial se realizó durante la semana previa al comienzo del estudio, donde se llevó a cabo una valoración antropométrica, un test de aproximación de una repetición máxima (RM) de cada uno de los ejercicios de musculación que posteriormente se ejecutaban en el entrenamiento experimental y un test de resistencia muscular local.

Se realizó una valoración antropométrica a través de un evaluador experimentado (nivel I de cineantropometría de la ISAK). Todas las valoraciones se llevaron a cabo a las 8.30 de la mańana en un lugar amplio y con temperatura de $18-20^{\circ} \mathrm{C}$. Los instrumentos de medida fueron calibrados antes de comenzar las mediciones y todas las medidas se tomaron en el lado derecho, aunque no fuese el dominante del deportista. Antes de comenzar con las mediciones se marcaron con lápiz demográfico los puntos anatómicos que sirvieron de referencia, y los instrumentos de medida se manipularon con la mano derecha.

En el registro de las medidas antropométricas (masa corporal y altura) y las correspondientes al cálculo del porcentaje de grasa corporal se empleó el protocolo propuesto por el Grupo Español de Cineantropometría (GREC) ${ }^{21}$ siguiendo la táctica de Rose y Guimares. La talla se obtuvo midiendo a los sujetos descalzos, de pie, con los talones, los glúteos y la espalda en contacto con la pared, con el tallímetro modelo Seca (Seca Ltd., Alemania). Para determinar el peso, los deportistas, descalzos y con ropa de deporte o interior, se colocaron de pie sobre la báscula eléctrica modelo Seca (Seca Ltd., Alemania). Se obtuvieron los pliegues cutáneos: tríceps, subescapular, bíceps, cresta ilíaca, supraespinal, abdominal, muslo frontal y pierna media, que se midieron por triplicado con el plicómetro Holtain Skinfold Caliper. Los perímetros corporales valorados fueron: brazo relajado, brazo contraído, cintura, cadera y pierna, y se midieron por triplicado utilizando la cinta métrica inextensible milimetrada de fibra de vidrio Holtain. Los diámetros valorados fueron: biestiloideo, biepicondíleo del húmero y bicondíleo del fémur, que se midieron por triplicado con el paquímetro Holtain.

Los datos se introdujeron en una plantilla de Excel elaborada por el ISAK, que directamente calculó los porcentajes de grasa, de masa muscular, de masa residual y de masa ósea.

A continuación, ese mismo día, se realizó una prueba de RM para 5 de los 10 ejercicios a testar. Para ello, los sujetos llevaban a cabo un pequeño calentamiento de ejercicio cardiovascular de 5 min de duración, más un calentamiento específico del grupo muscular a testar. Debían formalizar 3 series de calentamiento previas al test máximo. La primera serie constaba de 12 repeticiones con una carga baja, la cual podían movilizar con facilidad al menos 20 veces. La segunda serie era con una carga mayor, con la que pudiesen ejecutar 12-14 repeticiones, para 8 repeticiones. Y la tercera serie fue con una carga elevada, donde pudiesen movilizar la carga al menos 6 veces, movilizándose 4 repeticiones. Tras ello, se realizó el test de RM con una carga que el sujeto no pudiese desplazar más de 4 veces. Entre las series 1 y 2 y las 2 y 3 se mantuvo una recuperación de 2 min. Entre la serie 3 y el test de RM se mantuvo una recuperación de 3 min.

Pasadas 48 h de recuperación, se realizó un test de RM de los 5 ejercicios restantes, respetando exactamente el mismo protocolo. Una vez obtenidos los RM de cada ejercicio, se calculó el 60\% de 1RM del ejercicio de press de banca horizontal con barra y se llevó a cabo la evaluación de la resistencia muscular local en dicho ejercicio. Para ello se solicitó que el ejecutante hiciera tantas repeticiones correctas como le fuese posible hasta llegar al fallo muscular.

\section{Protocolo de entrenamiento}

A partir de este momento, los sujetos realizaron un protocolo de entrenamiento de fuerza usando el método "circuito intermitente con sobrecargas" a lo largo de 8 semanas, en las que se tuvieron que ejercitar 3 veces por semana, en días alternos (48 h de recuperación). Este método "híbrido" se puede definir como la unión del entrenamiento en circuito o circuit weight-training creado por Morgan y Adamson en $1952^{19,22}$ y el entrenamiento intermitente creado por Astrand en la década de los sesenta ${ }^{23}$. El entrenamiento consistió en la realización de un gran volumen de trabajo con 4 ejercicios de musculación de carácter global. Los sujetos llevaban a cabo 3 repeticiones de cada ejercicio, con una duración aproximada de $8 \mathrm{~s}$ de trabajo 
muscular, siguiendo la misma estructura de trabajo continuo que el entrenamiento en circuito pero usando una micropausa de $10 \mathrm{~s}$. Por tanto, se dio un ratio de 1:1,2 (8 s de trabajo muscular y $10 \mathrm{~s}$ recuperación). Según la planificación, las cargas fueron estimadas sobre el $62 \%$ de 1 RM de cada sujeto y, según se desarrolló el programa de entrenamiento, evolucionaron hasta el $72 \%$ RM. El volumen de entrenamiento comenzó en 24 series o vueltas y aumentó hasta llegar a un máximo de 36 series o vueltas. La duración del entrenamiento fue de 30 min en su inicio y evolucionó hasta el final del programa a $42 \mathrm{~min}$.

Durante el programa de entrenamiento de fuerza se controló la ingesta calórica de cada sujeto mediante un diario nutricional donde los sujetos experimentales anotaban el tipo de ingesta que hacían a lo largo del día, así como las cantidades ingeridas de cada alimento con el fin de conocer la cantidad de calorías ingeridas por cada uno.

Una vez finalizadas las 8 semanas de entrenamiento se procedió a la valoración final, que consistió en una valoración antropométrica y la estimación de $1 \mathrm{RM}$ respetando y siguiendo los mismos protocolos que en la valoración inicial del estudio.

Para el análisis de los datos se utilizó el paquete informático SPSS versión 14.0. Se realizó una prueba no paramétrica de Wilcoxon para 2 variables relacionadas con el fin de conocer diferencias significativas entre los resultados del pre y post-test de cada una de las variables objeto de estudio y se mantuvo como valor significativo $\mathrm{p}<0,05$. A su vez, se realizó un análisis de varianza de medidas repetidas para ver el efecto del tiempo en una variable, estableciendo significación estadística cuando $\mathrm{p}<0,05$.

\section{Resultados}

La tabla II muestra las mejoras obtenidas por el grupo de sujetos en composición corporal tras las 8 semanas de entrenamiento. En ella se describen las adaptaciones en la masa muscular $(+2,66 \%)$, el peso $(-2,04 \%)$, la masa grasa $(-1,4 \%)$ y la grasa corporal $(-9,46 \%)$

En la tabla III se observa la ingesta calórica de cada individuo a lo largo de las 8 semanas de duración del programa experimental.

La tabla IV se refiere a las mejoras producidas por el entrenamiento sobre la fuerza máxima $(3,61-21,31 \%)$ y el test de resistencia muscular local (11,42\%).

\section{Dıscusı́ón}

Los resultados obtenidos muestran que 8 semanas de entrenamiento de la fuerza utilizando un método de entrenamiento en circuito intermitente con sobrecargas tienen un efecto eficaz frente a la fuerza máxima, la resistencia muscular local y la composición corporal de los 8 sujetos expertos seleccionados.

Respecto a los parámetros antropométricos, se observa un descenso del peso corporal de 2,04\%, lo que concuerda con otros estudios que valoran los cambios en este criterio usando

Tabla II

Resultados en el pretest y el post-test en cuanto a composición corporal

\begin{tabular}{|lcccc|}
\hline & Pre-test & Post-test & Variación (\%) & Significación \\
\hline Peso & $78,71 \pm 7,3 \mathrm{~kg}$ & $77,10 \pm 6,5 \mathrm{~kg}$ & $-2,04$ & $*$ \\
\hline Grasa $(\%)$ & $18,06 \pm 3,6 \%$ & $16,35 \pm 3,6 \%$ & $-9,46$ & $*$ \\
\hline Masa grasa & $14,28 \pm 4,28 \mathrm{~kg}$ & $14,08 \pm 4,04 \mathrm{~kg}$ & $-1,4$ & $\mathrm{NS}$ \\
\hline Masa muscular & $35,59 \pm 2,24 \mathrm{~kg}$ & $36,54 \pm 2,5 \mathrm{~kg}$ & $+2,66$ \\
\hline
\end{tabular}

*Cambio significativo, $\mathrm{p}<0,05$; NS: cambio no significativo ( $>>0,05$ ); +: aumento; -: disminución.

Tabla III Ingesta calórica durante el programa de entrenamiento

\begin{tabular}{|lcccc|}
\hline Sujetos & Semana I y $\mathbf{2}$ (kcal) & Semana $\mathbf{3}$ y $\mathbf{4}$ (kcal) & Semana $\mathbf{5}$ y $\mathbf{6}$ (kcal) & Semana $\mathbf{7}$ y $\mathbf{8}$ (kcal) \\
\hline $\mathrm{n}=8$ & $2.775,00 \pm 173,20$ & $2.857,08 \pm I 42,59$ & $2.956,66 \pm 175,5 I^{*}$ & $2.833,67 \pm 208,80$ \\
\hline
\end{tabular}

*Cambio significativo en las semanas 5 y 6 respecto a la semana 1 y 2 . 
TRABAJOS ORIGINALES

Tabla IV

Resultados y mejoras en el pre y post-test de IRM y el test de resistencia muscular local

\begin{tabular}{|c|c|c|c|c|}
\hline Ejercicio & Pre-test (kg) & Post-test (kg) & Variación (\%) & Significación \\
\hline Press banca horizontal & $92,67 \pm 8,21$ & $96,52 \pm 7,14$ & $+4,15$ & $*$ \\
\hline Press banca declinado & $93,91 \pm 8,24$ & $98,22 \pm 7,35$ & $+6,43$ & NS \\
\hline Press banca inclinado & $80,75 \pm 8,77$ & $85,95 \pm 9,36$ & $+4,58$ & NS \\
\hline Prensa inclinada & $243,52 \pm 32,8$ & $294,55 \pm 51,09$ & $+20,95$ & $*$ \\
\hline Sentadilla multipower & $83,71 \pm 19,4$ & $101,55 \pm 21,86$ & $+21,31$ & $*$ \\
\hline Jalón frontal neutro & $104,97 \pm|4,9|$ & $109,02 \pm 15,50$ & $+3,85$ & NS \\
\hline Remo máquina & $110,74 \pm 14,69$ & $117,78 \pm 18,29$ & $+6,35$ & NS \\
\hline Curl bíceps barra Z & $51,14 \pm 5,69$ & $53,03 \pm 4,95$ & $+3,69$ & $*$ \\
\hline Extensiones codo polea alta & $87,81 \pm 4,71$ & $90,98 \pm 6,97$ & $+3,61$ & NS \\
\hline Remo vertical barra EZ & $49,51 \pm 4,52$ & $51,88 \pm 3,95$ & $+4,78$ & $*$ \\
\hline Resistencia muscular local & $20,7 \mathrm{I} \pm 2,2$ rep. & $23,12 \pm 1,64$ rep. & $+11,42$ & $*$ \\
\hline
\end{tabular}

*Cambio significativo, $\mathrm{p}<0,05$; NS: cambio no significativo; rep: repeticiones realizadas.

métodos de entrenamiento en circuito ${ }^{10,13,15,16,24}$. Dentro de la composición corporal, dos de los parámetros que más llaman la atención son el porcentaje de grasa corporal y el porcentaje de masa muscular. El incremento mostrado en la masa muscular está en torno al 2,66\%, mientras que la masa grasa desciende en torno al 1,4\%. Ambos valores son estadísticamente significativos, posiblemente por los pequeños valores de intervalo debido al tamaño de la muestra, pero dan una orientación positiva de la eficacia frente a los parámetros de este método de entrenamiento. Donde sí aparece una mejora significativa es en el porcentaje de grasa corporal, con una mejora del 9,46\%. Estos valores superan los resultados obtenidos por otros autores en cuanto al entrenamiento en circuito (circuit weight-training), los cuales obtienen disminuciones comprendidas entre el 0,38 y el $4,3 \%{ }^{10,11,13,16-19,22,25}$.

Estas claras mejoras se pueden deber a varios motivos. La ingesta calórica fue controlada, y aunque el objetivo era solamente conocer la cantidad de calorías que se ingerían a lo largo del estudio, sí podría haber influido en los sujetos como motivación. No obstante, se observó que los incrementos de la ingesta calórica coincidían con los incrementos en la carga de entrenamiento, lo que da mayor validez a estos resultados. De hecho, la mejora tan pronunciada en estos datos puede deberse al tipo de entrenamiento, ya que este circuito intermitente con sobrecargas produce una solicitación de las grasas como fuente de energía ${ }^{2}$. Al ser un tipo de entrenamiento de fuerza con tiempos de trabajo y de pausa más pequeños, que pone de ma- nifiesto una exigencia cardíaca mayor y una duración comprendida entre los 30 y los $42 \mathrm{~min}$, con una intensidad moderada, es lógico pensar en que se está produciendo esta solicitación de las grasas como sustrato energético.

Los porcentajes de mejora en la grasa corporal en los estudios analizados eran para individuos desentrenados, y el hecho de que en este estudio marcaran una diferencia bastante acusada podría deberse a la gran carga de entrenamiento en cuanto a intensidad y a volumen gracias a la experticia de la muestra y a la implicación de los grupos musculares que se ponen en marcha. Esto, unido a que es un trabajo continuo donde se busca una estabilización de la frecuencia cardíaca de trabajo, hace que el gasto calórico del entrenamiento se presuma elevado. Sin embargo, haría falta realizar un análisis de la energía requerida por el entrenamiento y la energía ingerida por los sujetos experimentales, para así obtener datos mucho más ajustados.

Otras adaptaciones de interés son las relacionadas con el incremento de la fuerza y de la resistencia muscular local. Si bien el desarrollo y la ganancia de la fuerza se han demostrado realizando métodos de entrenamiento estrictamente de fuerza, también se pueden obtener ganancias en esta capacidad usando métodos combinados de fuerza y de resistencia ${ }^{26}$. El entrenamiento en circuito con sobrecargas (circuit weight-training) ha demostrado producir grandes beneficios en esta capacidad, con mejoras de entre el 15 y el $42 \%^{11-13}$. Si bien estas mejoras son similares e incluso superiores a las obtenidas por nuestro méto- 
do de entrenamiento (entre 3,61 y 21,31\%), cabe diferenciar que la muestra seleccionada por los autores antes mencionados no poseían ningún tipo de experiencia en el entrenamiento con sobrecargas.

Es conocido que la fuerza muscular en hombres y mujeres sanos, previamente desentrenados, puede aumentar siempre que las cargas de entrenamiento superen suficientemente las actividades normales diarias de un músculo en particular ${ }^{27}$; por tanto, cualquier método que use sobrecargas que superen dicha intensidad puede provocar grandes mejoras en la fuerza de los sujetos desentrenados. Cargas cercanas al 50\% del RM producen mejoras en la fuerza máxima en personas desentrenadas, pero los sujetos que ya han adquirido cierto grado de práctica en el entrenamiento con sobrecarga requieren intensidades superiores ( $>60 \%$ de $1 \mathrm{RM}$ ) para obtener adaptaciones significativas $^{28}$. Sin embargo, la muestra seleccionada para el presente estudio se basó en expertos en dicho entrenamiento ${ }^{20}$, por lo que las adaptaciones obtenidas llaman la atención, ya que los sujetos estaban acostumbrados al levantamiento de cargas más pesadas.

Tal vez una de las explicaciones que deriva de estas adaptaciones pueda ser que, después del programa experimental, los sujetos entrenaron 3 sesiones por semana los mismos grupos musculares, por lo que triplicaron el número de entrenamientos que estaban acostumbrados a realizar. Debido a este cambio en sus entrenamientos, las mejoras tras las 8 semanas de programa experimental se dieron con la utilización de cargas moderadas.
Por último, se muestran incrementos significativos en cuanto a la resistencia muscular local en torno al 11,42\% (tabla III). Esta mejora es un poco más evidente que la mejora en cuanto a la fuerza dinámica máxima, ya que consiste en ejecutar el mayor número de repeticiones a una intensidad del $60 \%$, situándose la intensidad desarrollada a lo largo del programa de entrenamiento en torno a este valor. Es lógico pensar que existan adaptaciones a nivel muscular, tendinoso, ligamentoso y articular para responder a las demandas de un entrenamiento donde el trabajo muscular es constante, debido a que los 4 ejercicios se repiten entre 24 y 36 veces a lo largo de una misma sesión, con lo cual no sólo la exigencia cardíaca es patente, sino también la muscular. Aunque no existen datos sobre este método de entrenamiento, por ser inédito, es bien sabido que este tipo de adaptaciones musculares se dan en programas de entrenamiento en circuito ${ }^{11,12}$.

\section{Conclusiones}

El entrenamiento en circuito intermitente con sobrecargas de 8 semanas de duración ofrece adaptaciones en cuanto a la composición corporal, la fuerza máxima y la resistencia muscular local en sujetos entrenados en musculación.

El entrenamiento en circuito intermitente puede resultar un método alternativo y eficaz para producir mejoras en la composición corporal mediante ejercicios con sobrecargas, y se requiere la realización de más estudios con muestras más amplias y en diferentes poblaciones.

\section{Bibliografía}

1. Brandenburg J, Docherty D. The effect of training volume on the acute response and adaptations to resistance training. International Journal of Sports Physiology and Performance. 2006;1:10821.

2. Ahtiainen JP, Pakarinen A, Alen M, Kraemer W, Hakkinen K. Short vs. long rest period between the sets in hypertrophic resistance training: influence on muscle strength, size, and hormonal adaptations in trained men. J Strength Cond Res. 2005;19:57282.

3. Jonathan P, Williams F, Williams AG. The adaptations to strength training morphological and neurological contributions to increased strength. Sports Medicine. 2007;37:145-68.

4. Becerro M. Entrenamiento de fuerza para todos. Federación Internacional de Halterofilia. 2000.
5. Warburton DE, Gledhill N, Quinney A. Musculoskeletal fitness and health. Can J Appl Physiol. 2001;26:217-37.

6. Bird SP, Tarpenning KM, Marino FE. Designing resistance training programmes to enhance muscular fitness a review of the acute programme variables. Sports Medicine. 2005;35:841-51.

7. Sakamoto A, Sinclair P. Effect of movement velocity on the relationship between training load and the number of repetitions of bench press. J Strength Cond Res. 2006;20:523-7.

8. Spreuwenberg L, Kraemer WJ, Spiering BA, Volek JS, Hatfield $\mathrm{DL}$, Silvestre R, et al. Influence of exercise order in a resistancetraining exercise session. J Strength Cond Res. 2006;20:141-4.

9. Wolfe BL, LeMura LM, Cole P. Quantitative analysis of singlevs. multiple-set programs in resistance training. J Strength Cond Res. 2004;18:35-47. 
10. Gettman LR, Ward P, Hagan RD. A comparison of combined running and weight training with circuit weight training. Medicine Science Sports Exercise. 1982;14:229-34.

11. Kaikkonen H, Yrjama M, Siljander E, Byman P, Laukkamen R. The effects of heart rate controlled low resistance circuit weight training and endurance training on maximal aerobic power in sedentary adults. Scand J Med Sci Sports. 2000;10:211-5.

12. Harber MP, Fry AC, Rubin MR, Smith JC, Weiss LW. Skeletal muscles and hormonal adaptations to circuit weight training in untrained men. Scand J Med Sci Sports. 2004;14:176-85.

13. Mosher PE, Underwood SA, Ferguson MA, Arnold RO. Effects of 12 weeks of aerobic circuit training on aerobic capacity, muscular strength and body composition in collage-women. J Strength Cond Res. 1994;8:144-8.

14. Wilmore JH, Parr RB, Girandola RN, Ward P, Vodak PA, Barstow TJ, et al. Physiological alterations consequent to circuit weight training. Medicine Science Sports. 1978;10:79-84.

15. Garbutt G, Boocock MG, Reilly T, Troup DG. Physiological and spinal responses to circuit weight training. 1994; 4:46-9.

16. Gettman LR, Pollock ML. Circuit weight training: A critical review of its physiological benefits. Physician \& Sports Medicine. 1981;9:44-9.

17. Gotshalk L, Berger R, Kraemer W. Cardiovascular responses to high-volume continuous circuit resistance training protocol. J Strength Cond Res. 2004;18:760-4.

18. Halton RW, Kraemer RR, Sloan A, Hebert EP, Frank K, Trniecki JL. Circuit weight training and its effects on excess post exercise oxygen consumption. Med Sci Sports Exerc. 1999;31:1613:8.
19. Tous J. Nuevas tendencias en fuerza y musculación. Barcelona: Ergo; 1999.

20. Carpinelli R, Otto R, Winett R. A critical analysis of the ACSM Position Stand on resistance training: insufficient evidence to support recommended training protocols. Journal of Exercise Physiology online. 2004;7:3.

21. Esparza, F. Manual de cineantropometría. Monografías Femede. 1993.

22. Colado JC. Fitness en la sala de musculación. Barcelona: Inde; 1996.

23. Astrand I, Astrand PO, Christensen EH, et al. Intermittent muscular work. Acta Physiol Scand. 1960;48:448-53.

24. Harris KA, Holly RG. Physiological response to circuit weight training in borderline hypertensive subjects. Med Sci Sports Exerc. 1987;19:246-52.

25. McCarthy JP, Agre JC, Graf BK, Pozniak MA, Vailas AC. Compatibility of adaptative responses with combining strength and endurance training. Medicine Science \& Sports Exercise. 1995;27: 429-36.

26. Warburton DE, Gledhill N, Quinney A. The effects of changes in musculoskeletal fitness on health. Can J Appl Physiol. 2001;26: 161-216.

27. Häkkinen K. Adaptación neuromuscular al entrenamiento de la fuerza en hombres y mujeres. Resúmenes del 1 . $^{\text {er }}$ Simposio Internacional de Fuerza y Potencia relacionadas con los Deportes, la actividad Física, el Fitness y la Rehabilitación. 2000.

28. Hass CJ, Feigenbaum MS, Franklin BA. Prescription of resistance training for healthy population. Sports Medicine. 2001;31:953-64. 